\title{
Derivative optical spectroscopy of thin films of alkaline-earth titanates: Critical points
}

Jarmila Müllerová, Pavel Šutta, and Lucie Prušáková

Citation: AIP Conference Proceedings 1996, 020030 (2018); doi: 10.1063/1.5048882

View online: https://doi.org/10.1063/1.5048882

View Table of Contents: http://aip.scitation.org/toc/apc/1996/1

Published by the American Institute of Physics

\section{Articles you may be interested in}

Criticality safety validation of the SCALE system

AIP Conference Proceedings 1996, 020029 (2018); 10.1063/1.5048881

Optimization of X-ray imaging by Timepix based radiation camera with SI GaAs sensor

AIP Conference Proceedings 1996, 020039 (2018); 10.1063/1.5048891

Properties of nanocrystalline alloys after electron beam irradiation of amorphous precursor

AIP Conference Proceedings 1996, 020043 (2018); 10.1063/1.5048895

Design of low loss silicon nitride 8-channel AWG

AIP Conference Proceedings 1996, 020042 (2018); 10.1063/1.5048894

Monitoring of PET cyclotron radiation fields using a novel Bonner sphere spectrometer

AIP Conference Proceedings 1996, 020037 (2018); 10.1063/1.5048889

XPS limit in soft X-ray photoemission spectroscopy of $\mathrm{Ag}(001)$

AIP Conference Proceedings 1996, 020033 (2018); 10.1063/1.5048885

\section{Conference Proceedings}

\section{Get $30 \%$ off all print proceedings!}

Enter Promotion Code PDF30 at checkout 


\title{
Derivative Optical Spectroscopy of Thin Films of Alkaline- Earth Titanates: Critical Points
}

\author{
Jarmila Müllerová $^{1, a)}$, Pavel Šutta ${ }^{2, b)}$ and Lucie Prušáková, 2 c) \\ ${ }^{1}$ Institute of Aurel Stodola, Faculty of Electrical Engineering, University of Žilina \\ ul. kpt. J. Nálepku 1390, 03101 Liptovský Mikuláš, Slovak Republic \\ ${ }^{2}$ New Technologies - Research Centre, University of West Bohemia \\ Univerzitní 8, 30614 Plzeň, Czech Republic \\ ${ }^{a)}$ Corresponding authors: mullerova@1m.uniza.sk, ${ }^{\text {b) }}$ sutta@ntc.zcu.cz, ${ }^{\text {c) }} 1$ prusak@ntc.zcu.cz
}

\begin{abstract}
This paper analyzes UV Vis absorption spectra of amorphous thin films of barium strontium titanate (BST) deposited by RF magnetron sputtering on $\mathrm{SiO}_{2}$ at different substrate temperatures. The Tauc procedure was used to determine optical band gaps for direct and indirect interband transitions. This procedure was accompanied by derivative spectroscopy useful in case of multicomponent systems. Higher-order derivatives were used to find the so-called critical points related to the optical interband transitions at various regions in the Brillouin zone. Three critical points were identified in BST thin films spectra of dielectric function. All optical band gaps and energies of critical points were found to decrease with the substrate temperature at the deposition.
\end{abstract}

\section{INTRODUCTION}

Alkaline earth titanates such as $\mathrm{BaTiO}_{3}(\mathrm{BTO}), \mathrm{SrTiO}_{3}(\mathrm{STO})$ are perovskite-type compounds with excellent properties suitable for electronic and optoelectronic devices, e.g. as sensors, actuators, optical modulators, optical windows and memories, optical filters etc. [1], [2]. The same octahedral coordination provides similar properties of BTO and STO. Nevertheless properties differ substantially in dependence on structure, microstructure and composition and therefore continuing research is welcome. Wide-band gap BTO and STO can well accommodate different dopants that can influence not only electric properties but also the optical response. Barium substituted STO $\left(\mathrm{Ba}_{\mathrm{x}} \mathrm{Sr}_{1-\mathrm{x}} \mathrm{TiO}_{3}, \mathrm{BST}\right)$ is a ferroelectric material of good thermal stability used in non-volatile memories, dielectric capacitors etc. Refractive and absorptive optical properties important for all light-based applications are reported as compositionally activated [3] - [7].

Absorption peaks in UV Vis region of multicomponent materials are normally broad due to overlaps of many optical transitions. Usually a common practice to analyze the absorption spectrum is reduced to the determination of the absorption edges or optical band gaps via procedures described in [8], all of them based on some compromises. For most light-based applications this approach is fully sufficient but does not bring any additional experimental knowledge on spectral components contributing to the optical interband transitions in a specific material. However overall absorption of a compound includes absorption features of all particular components.

The mostly applied method of analysis of particular absorption features is spectral decomposition. The decomposition into absorption components is often used when there is an indicative of an asymmetric absorption peak. However particular absorption features corresponding to interband optical transitions are often complicated to resolve. Derivative spectroscopy is a commonly used technique that enables to resolve the overlapping and nearby lying absorption peaks in case of a multicomponent material that cannot be easily identified in the original absorption spectrum $[9,10]$. The method uses higher-order derivatives of absorbance or dielectric function with respect to the wavelength or photon energy. Derivative spectroscopy is mainly aimed at quantitative chemical analysis of organic materials or biological tissues $[11,12]$. The main idea is that the parent zero-order spectrum of

Applied Physics of Condensed Matter (APCOM 2018)

AIP Conf. Proc. 1996, 020030-1-020030-6; https://doi.org/10.1063/1.5048882

Published by AIP Publishing. 978-0-7354-1712-0/\$30.00 
the absorbance or the dielectric function undergoes a significant change after converting into higher-order derivatives.

Derivative spectroscopy in the absorption analysis of metals, inorganic and hybrid semiconductors and their structures is less frequent $[13,14]$ although it brings additional knowledge on multicomponent compounds or on o materials under stress conditions $[15,16]$. In this paper we report on resolution enhancement of the determination of optical interband transitions using derivatives of higher orders. A series of five thin films of BST deposited by RF magnetron sputtering on glass served as a featured example.

\section{EXPERIMENTAL}

A series of thin films of quaternary $\mathrm{Ba}_{\mathrm{x}} \mathrm{Sr}_{1-\mathrm{x}} \mathrm{TiO}_{3}$ (BST, samples 21 - 25) were deposited on $\mathrm{SiO}_{2}$ substrate by RF magnetron sputtering (13.56 MHz radio frequency) using BOC Edwards TF 600 coating system from the cleaned deposition target $\left(\mathrm{Ba}_{0.1} \mathrm{Sr}_{0.9} \mathrm{TiO}_{3} 3\right.$ inch target, purity 99.9 at.\%). The deposition was kept under constant discharge $\mathrm{RF}$ power of $400 \mathrm{~W}$. The samples $21-23$ were deposited in argon atmosphere at $5 \mathrm{sccm}$ at constant pressure of 0.6 $\mathrm{Pa}$. The samples 24 and 25 were deposited in mixed argon $(5 \mathrm{sccm})$ and oxygen $(0.25 \mathrm{sccm})$ atmosphere. The thickness of the films measured by Kla Tencor Profilometer was $\sim 300 \mathrm{~nm}$. The influence of the substrate temperature was evaluated. The substrate temperature was room temperature (samples 21 and 24), $100{ }^{\circ} \mathrm{C}(22)$ and $400{ }^{\circ} \mathrm{C}$ (samples 23 and 25). No exact knowledge on $\mathrm{Ba}$ to $\mathrm{Sr}$ content in thin films is available. However as the deposition was performed from a single target with highly prevailing $\mathrm{Sr} / \mathrm{Ba}$ ratio $=0.9 / 0.1$ this dominance can be predicted in the deposited films.

XRD patterns of the samples deposited on $\mathrm{SiO}_{2}$ and $\mathrm{Si}$ wafers taken by an automatic powder diffractometer XPert Pro equipped with a proportional point detector and $\mathrm{CuK} \alpha$ radiation as an $\mathrm{X}$-ray source were detected the samples being amorphous. Optical transmittances of the samples deposited on $\mathrm{SiO}_{2}$ were recorded at nearly normal incidence in wide spectral range of $(190-1100) \mathrm{nm}$ by Specord 210 spectrophotometer with air blank reference channel. In the optical transmittance spectra (Fig. 1) apparent interference fringes are present due to weak absorption of light above the absorption edge. Different onsets of the material transparency indicate the differences in optical band gaps of the samples deposited under different deposition substrate temperature.

\section{TRANSMITTANCE SPECTRA ANALYSIS: OPTICAL PROPERTIES}

The optical transmittance of a homogeneous non-scattering thin film with parallel interfaces deposited on a thick uncoherent substrate is a nonlinear function of the wavelength, the film thickness and the complex refractive indices $N=n+i k$ of the film and the substrate (real and imaginary parts are the refractive index $n$ and the extinction coefficient $k$ ). For the samples under study spectral $n$ and $k$ were extracted from measured transmittance spectra using a global optimization procedure based on genetic algorithm. The theoretical transmittance was calculated using the theory in [8] and the Tauc-Lorentz dispersion model for the dependence of $n, k$ on the wavelength [17]. Resulting spectra of $n, k$ are in Fig. 2. Fig. 3 shows the spectrum of the absorption coefficient $\alpha$ that is related to $k$ and the wavelength $\lambda$ as $\alpha=4 \pi k / \lambda$. An additional outcome from the transmittance spectra fitting is the film thickness mentioned in Table 1.

A meaningful procedure to achieve knowledge on the optical band gaps is the inspection of the absorption spectrum via a usual practice called the Tauc plot. Due to disorder in amorphous materials the valence and conduction band edges are extended with no clear abrupt energy above which photons can be absorbed via interband transitions. Therefore the optical band gaps cannot been determined unambiguously and are usually resolved from the extrapolation of linear parts of the so-called Tauc plots $\alpha E_{\text {phot }} \sim\left(E_{p h o t}-E_{g}\right)^{n}$ (where the photon energy $E_{p h o t}=h c / \lambda, h$ is the Planck's constant, $c$ is the speed of light, $E_{g}$ is the Tauc optical band gap) to the energy axis. The transition order is $n=1 / 2$ (2) for direct (indirect) interband transitions. Optical band gaps of BST determined using this procedure are listed in Table 1 . We see a slight decrease with increasing substrate temperature which can be recognized for both direct and indirect transitions. The fundamental interband absorption is here shifted to longer wavelengths. $E_{g}$ values summarized in Table 1 are higher than reported for STO and BST with prevailing strontium content $[15,18]$.

It must be emphasized that linear parts of the Tauc plots of all samples were reduced to some extent. This obstacle caused certain ambiguities in determining optical band gaps. Therefore we used the so-called iso-absorption gap energy $E_{04}$ as an additional figure of merit to review the absorption onset. $E_{04}$ is the photon energy at which the absorption coefficient achieves the value of $10^{4} \mathrm{~cm}^{-1}$. Although $E_{04}$ is not reflecting particular differences in optical 
absorption it is tolerable especially in the presence of the continuous distribution of localized states between the valence and conduction bands. The values of $E_{04}$ in Table 1 follow the trend observed in $E_{g}$ namely the narrowing of the optical band gap with increasing substrate temperature.

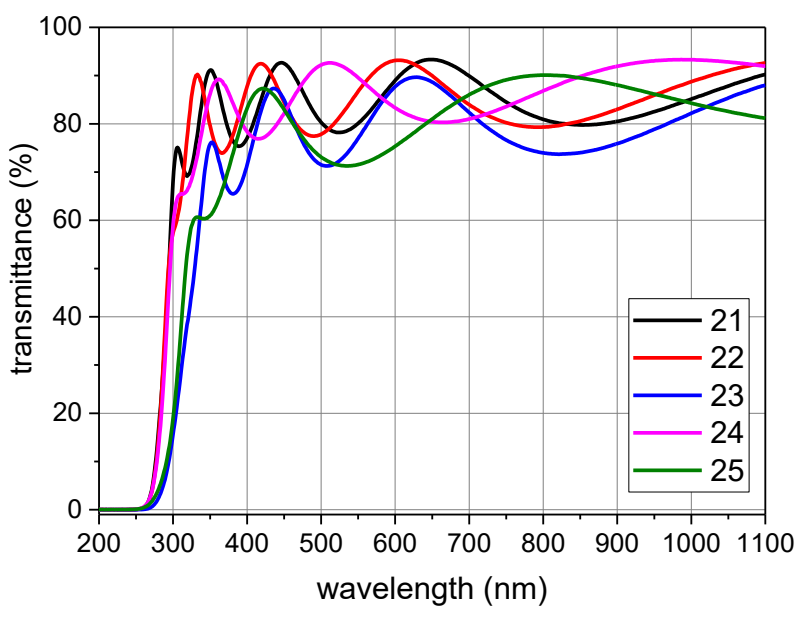

FIGURE 1. Transmittance spectra of the BST thin films under study.

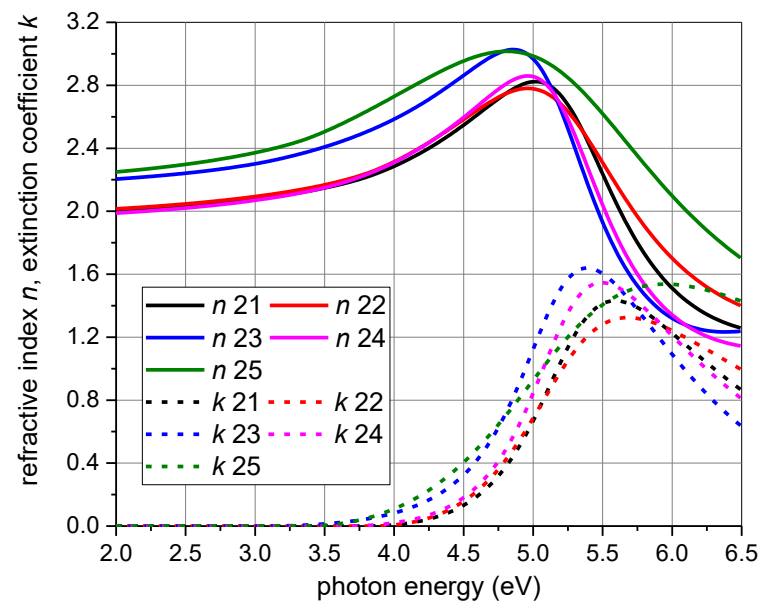

FIGURE 2. Refractive indices and extinction coefficients determined from the transmittance spectra.

\section{DIELECTRIC FUNCTION ANALYSIS: CRITICAL POINTS}

The macroscopic optical response of a material is represented by the dielectric function. The complex refractive index $N=n+i k$ is related to the dielectric function $\varepsilon=\varepsilon_{1}+i \varepsilon_{2}$ as $N^{2} \equiv \varepsilon$. The real and imaginary parts of the dielectric function are related to $n, k$ as $\varepsilon_{1}=n^{2}+k^{2}$ and $\varepsilon_{2}=2 n k$. They can be seen in Fig.4 as calculated from $n$, $k$ determined from the transmittance spectra. Spectra of $\varepsilon_{1}, \varepsilon_{2}$ were used as the parent zero-spectra for higher-order differentiations.

The experimental transmittance data were recorded with the $1 \mathrm{~nm}$-resolution in the wavelength axis. Therefore also the resolution of all subsequent calculated spectra is $1 \mathrm{~nm}$. As the obtained spectra were smooth and continuous no additional interpolation and smoothing was applied before differentiation.

The extremes in the dielectric function spectra, the so-called critical points (van Hove singularities) are attributed to optical interband transitions at various regions in the Brillouin zone [15]. In other words a critical point is a prominent feature in the dielectric function spectrum. Therefore information retrieved from the dielectric function spectrum is related to the band structure. Analysis of critical points of the interband transitions of electrons as prominent features in the optical response was developed by Cardona and others [19].

The higher-order derivatives of the zero-spectrum are often engaged in derivative spectroscopy to enhance the critical points structure. A maximum in the zero-spectrum is transformed into an inflection point in the second derivative and a minimum in the third derivative. As an example derivative spectra of the second and the fourth order of $\varepsilon_{1}$ with respect to the photon energies are shown in Fig. 5. The higher order of differentiation can be used for achieving higher discrimination of small features in the region $(3-4) \mathrm{eV}$. The reproduction of different parts of the derivative spectra (at $\sim 3.8 \mathrm{eV}$ and $5 \mathrm{eV}$ ) is not similar in the amplitudes of the derivatives. The higher the order of the derivative the more pronounced the region at $\sim 3.8 \mathrm{eV}$ becomes.

It is important to emphasize that the real $\varepsilon_{1}$ and the imaginary $\varepsilon_{2}$ parts of the dielectric function associated with the refraction and absorption processes are linked together through the Kramers-Kronig (K-K) relations. The K-K causality means that an extremum of $\varepsilon_{1}$ at a specific wavelength is accompanied with the inflection point in $\varepsilon_{2}$ at the same wavelength and vice versa. This causality was be applied to determine critical points from higher-order derivatives of $\varepsilon_{1}, \varepsilon_{2}$. 


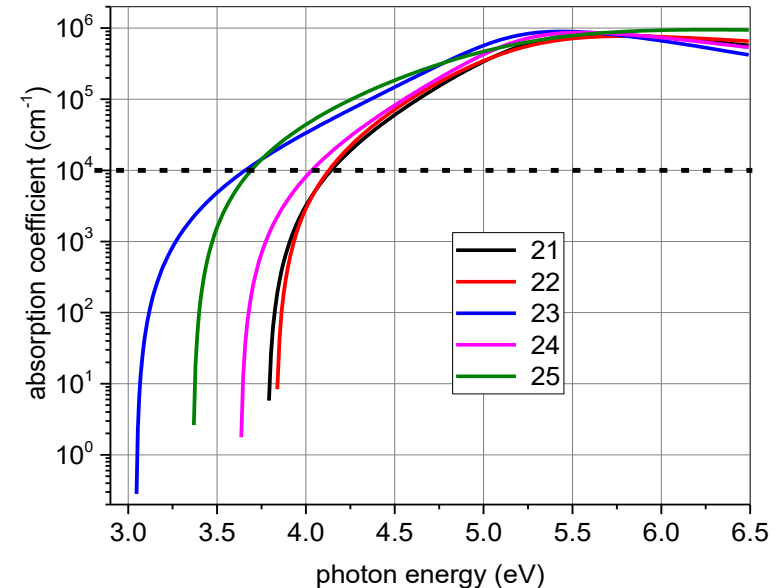

FIGURE 3. Absorption coefficients of BST thin films. The level of $E_{04}$ indicated.

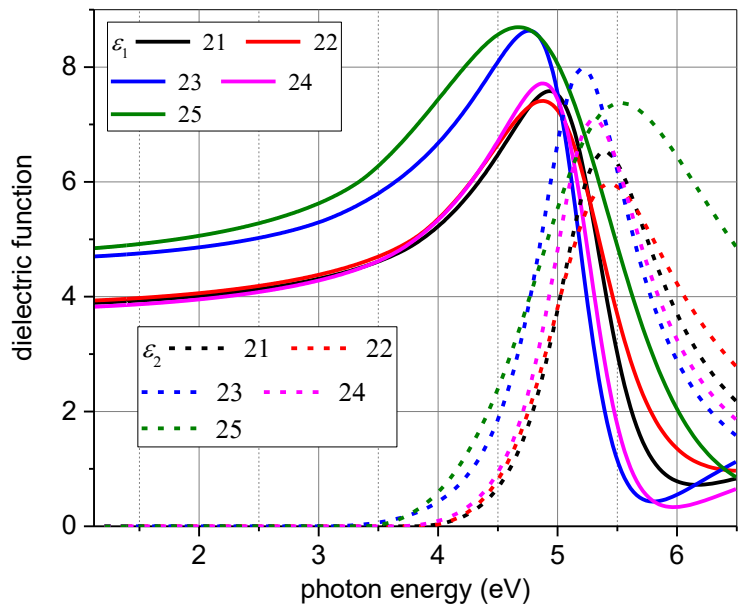

FIGURE 4. Dielectric functions of BST thin films, $\varepsilon_{1}$ solid line, $\varepsilon_{2}$ dash line.

For the determination of the critical points we use the third derivatives of real $\varepsilon_{1}$ and imaginary $\varepsilon_{2}$ part of the dielectric function executed separately. The third derivative spectra with respect to the photon energies are in Fig. 6. The critical points correspond to zero values of the third derivatives of $\varepsilon_{1}$ (inflection points) and maxima/minima of the third derivatives of $\varepsilon_{2}$. Three critical points were identified (Table 1). Critical points in the region of $(3-3.8) \mathrm{eV}$ cannot be straightforwardly revealed in the original absorption spectra (Fig. 3) and in the parent dielectric function (Fig. 4). For better interpretation this spectral region is depicted separately in Fig. 7.
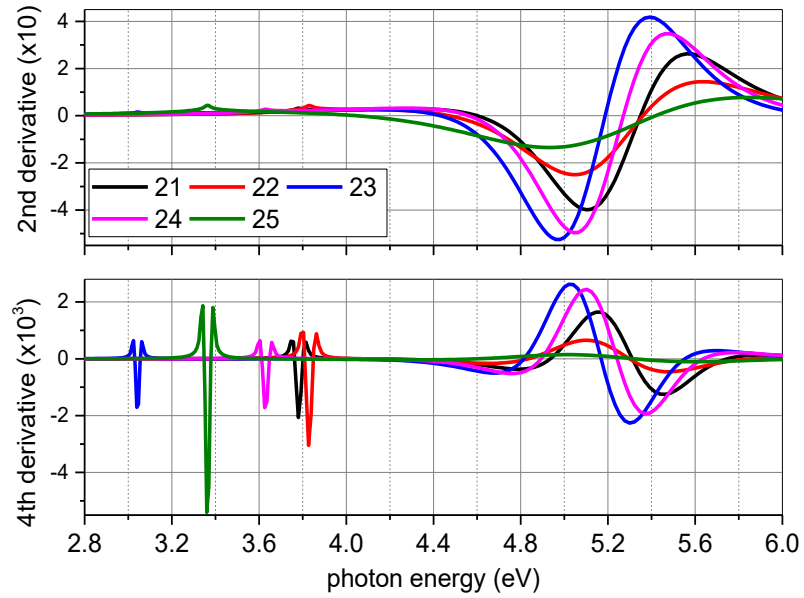

FIGURE 5. The comparison of the amplitudes of the second and the fourth derivatives of $\varepsilon_{1}$.

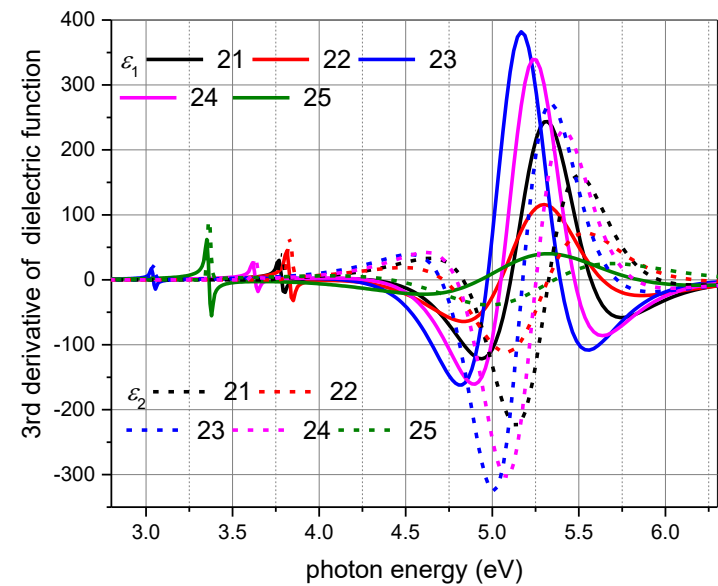

FIGURE 6. The third derivative of the dielectric function, $\varepsilon_{1}$ solid line, $\varepsilon_{2}$ dash line.

The positions of critical points obtained from the third derivative of $\varepsilon_{1}, \varepsilon_{2}$ are expected to coincide and in fact good agreement was achieved. The analysis of the critical points by the zero-crossing method of the third derivative of $\varepsilon_{1}$ is more sensitive to the photon energy than extrema identification in the third derivative of $\varepsilon_{2}$. Although the differences are less than $3 \%$ the values from the zero-crossing of the third derivatives of $\varepsilon_{1}$ were considered to be more accurate and are registered in Table 1.

The exact functional form of the dielectric function in the vicinity of the critical points was not the objective of this paper. The assignment of the critical point to optical interband transitions at certain points of the Brillouin zone 
is difficult. Partial coincidence of the critical points CP I and CP II with the critical points of STO can be observed [15]. Critical points at $\sim 5 \mathrm{eV}$ and $5.2 \mathrm{eV}$ are broad due to the disorder in amorphous thin films. The critical point at $\sim 3.8 \mathrm{eV}$ lies below the Tauc band gap for direct and indirect transition and its spectral width is significantly smaller than that of the other two identified critical points.

The decreasing tendency of Tauc optical band gaps with the substrate temperature coexists well also for critical points as can be seen in Table 1 and Fig. 8. This observation remains true for the samples deposited under pure argon atmosphere and also in mixed argon + oxygen atmosphere.

TABLE 1. Photon energies corresponding to the absorption features determined directly from the absorption spectra: $E_{04}$ and Tauc band gap energies $E_{\mathrm{g}}$ for direct and indirect interband transitions. Features assigned to critical points $(\mathrm{CP})$ determined

\begin{tabular}{cccccccc}
\hline Sample & $\begin{array}{c}\text { Thickness } \\
(\mathbf{n m})\end{array}$ & $\begin{array}{c}\boldsymbol{E}_{\mathbf{0 4}} \\
\mathbf{( e V )}\end{array}$ & $\boldsymbol{E}_{\boldsymbol{g}}$ indirect $(\mathbf{e V})$ & $\boldsymbol{E}_{\boldsymbol{g}} \mathbf{d i r e c t}(\mathbf{e V})$ & $\begin{array}{c}\text { CP I } \\
(\mathbf{e V})\end{array}$ & $\begin{array}{c}\text { CP II } \\
(\mathbf{e V})\end{array}$ & $\begin{array}{c}\text { CP III } \\
(\mathbf{e V})\end{array}$ \\
\hline 21 & 325 & 4.14 & 4.10 & 4.50 & 3.78 & 5.10 & 5.23 \\
22 & 300 & 4.13 & 4.05 & 4.43 & 3.83 & 5.04 & 5.19 \\
23 & 286 & 3.66 & 3.88 & 4.36 & 3.04 & 4.97 & 5.12 \\
24 & 254 & 4.03 & 4.10 & 4.50 & 3.62 & 5.05 & 5.19 \\
25 & 178 & 3.68 & 3.65 & 4.36 & 3.36 & 4.93 & 5.17 \\
\hline
\end{tabular}

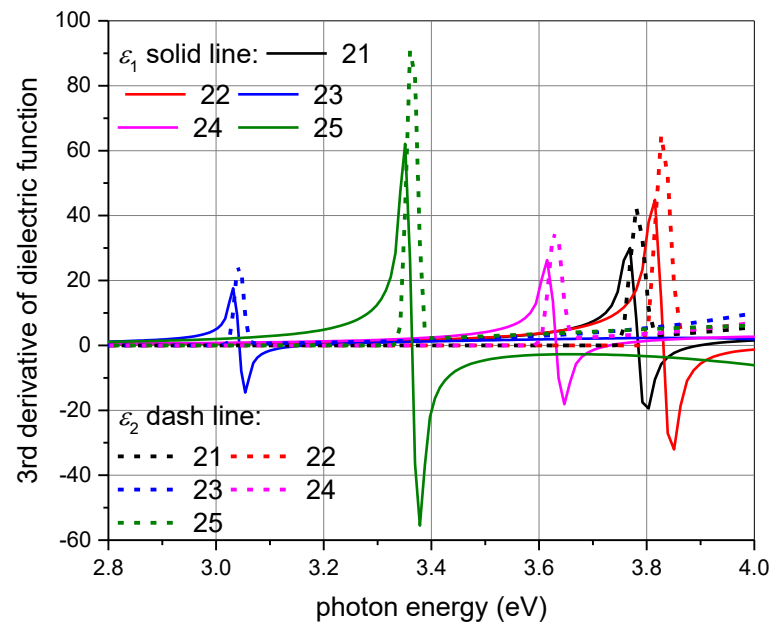

FIGURE 7. The third derivative of the real and imaginary parts of the dielectric function.

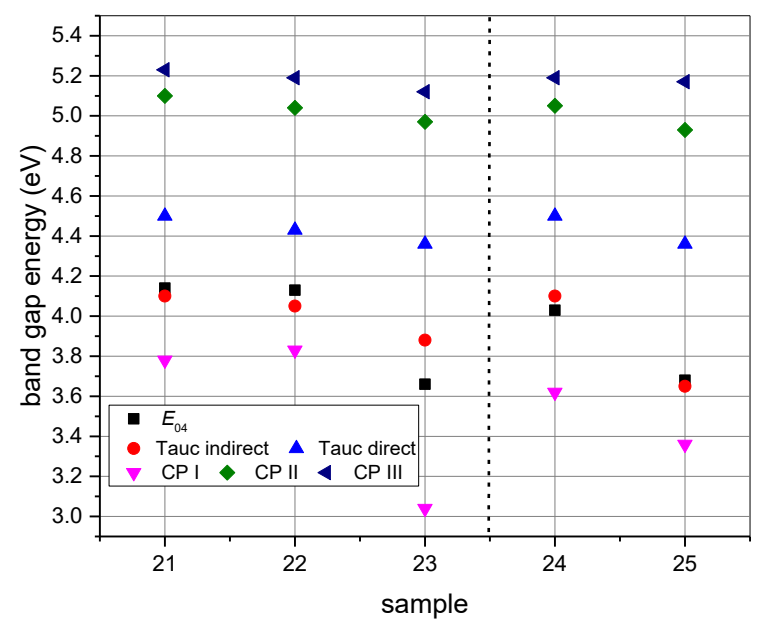

FIGURE 8. Critical points (CP), Tauc optical band gaps and isoabsorption energies $E_{04}$ for the samples $21-25$. The deposition differences explained in the part Experimental.

\section{CONCLUSIONS}

Derivative analysis of the dielectric function of BST thin films deposited by RF magnetron sputtering on $\mathrm{SiO}_{2}$ substrate was applied to determine critical points assigned to the optical interband transitions. The real and imaginary parts of the complex refractive index and of the dielectric function were determined from the transmittance spectra. The Kramers-Kronig causality was used to identify three critical points from the third derivative of the dielectric function. The positions of the critical points were correlated to the optical band gap energies for direct and indirect optical interband transitions determined from Tauc plots. All energy features contributing to the interband optical transitions were found to be sensitive to the substrate temperature used at the deposition process. 


\section{ACKNOWLEDGMENTS}

This work was partly supported by the Slovak Research and Development Agency under the projects APVV-15152, APVV-0888-11 and by the Slovak Grant Agency under the project VEGA 1/0840/18. The paper has received funding from the EU Horizon 2020 Research and Innovation Programme under the Marie Sklodowska-Curie grant agreement No. 734331 and by the Project CEDAMNF CZ.02.1.01./0/15_003/0000358 co-funded by the ERDF. This work occurred in frame of the LNSM infrastructure, project number LM 2015087 (MŠMT ČR).

\section{REFERENCES}

1. D. J. Kok, K. Irmscher, M. Naumann, Ch. Guguschev, Z. Galazka and R. Uecker, Phys. Stat. Sol. A 212, 1880-1887 (2015).

2. I.-D. Kim, Y. Avrahami, L. Socci, F. Lopez-Royo and H. L.Tuller, J. Asian Ceramic Soc. 2, 231-234 (2014).

3. G.S. Kathait, V. Rohilla, P. Thapliyal, D. Biswas and S. Singh, Int. J. Latest Technol. Eng. Manag. \& Appl. Sci. VI, 75-79 (2017).

4. H.Y.Tian, H.L.W. Chan, Ch.L. Choy and K. No, Mat. Sci. Eng. B103, 246-252 (2003).

5. M. Kocanda, S. F. Mohiudin and I.Abdel-Motaleb, Crys. Struct. Theory \& Appl. 1, 17-20 (2012).

6. J.Reséndiz-Muñoz, J.L. Fernández-Muñoz, M.A. Corona-Rivera, M. Zapata-Torres, A. Márquez-Herrera, M. Meléndez-Lira, F. Caballero-Briones, F. Chale-Lara and O. Zelaya-Ángel, J. Nanomat. 2017, Article ID 4308294, 8 pages (2017).

7. A.W. Nuayi, H. Alatas, I.S. Husein and M. Rahmat, Int. J. Optics 2014, Article ID 5314145, 8 pages (2014).

8. J. Müllerová and P. Šutta, Communications 19, 9-15, (2017).

9. M. Stolarczyk, A. Apola, A. Maślanka, A. Kwiecień and W. Opoka, Acta Pharm. 67, 463-478 (2017).

10. T.D. Kang, H. Lee, G. Xing, N. Izumskaya, V. Avrutin, B. Xiao and H. Morkoç, Appl. Phys. Lett. 91, 022918$1-3$ (2007).

11. A.-A. Y. El-Sayed and N. A. El-Salem, Anal. Sci. 21, 595-614 (2005).

12. C. Bosch Ojeda and F. Sanchez Rojas, Microchem. J. 106, 1-16 (2013).

13. H. Lee, J.-W. Park, H. Lee, Y.S. Park and H.-K. Kim, J. Korean Phys. Soc. 56, 625-632 (2010).

14. A. Varghese and A.M.A. Khadar, Acta Chim. Slov. 53, 374-380 (2006).

15. S. Zollner, A.A. Demkov, R. Liu, P.L. Fejes, R.B. Gregory, J.A. Curless, Z. Yu, J. Ramdani, R. Droopad, T.E. Tiwald, J.N. Hilfiker and J.A. Woollam, J. Vac. Sci. Technol. B18, 2242-2254 (2000).

16. C.J. Vineis, Phys. Rev. B 71, 245205-1-6 (2005).

17. G.E. Jellison Jr. and F.A. Modine, Appl. Phys. Lett. 69, 371-373 (1996), Erratum Appl. Phys. Lett. 69, 2137 (1996).

18. S.-H. Yang, D. Mo, H.-Y. Tian, W.-G. Luo, X.-H. Pu and A.-L. Ding, Phys. Stat Sol. A 191, 605-612 (2002).

19. S. Loughin, R.H. French, L.K. De Noyer, W.-Y. Ching and Y.-N. Xu, J. Phys. D: App. Phys. 29, 1740-1750 (1996). 